\title{
UNA LEY DE CONTRATOS PÚBLICOS PARA TIEMPOS DE CRISIS*
}

\author{
Francisco L. Hernández González \\ Profesor titular de Derecho Administrativo \\ Universidad de La Laguna
}

\section{RESUMEN}

El presente trabajo analiza cómo la legislación española de contratación pública ha podido afrontar las situaciones de crisis a lo largo de la historia, destacando las dificultades de la Ley 9/2017 para responder a la situación de crisis sanitaria y económica provocada por la covid-19. Es necesaria una contratación pública estratégica con capacidad para afrontar situaciones futuras de crisis.

Palabras clave: contratos públicos, crisis sanitaria y económica, covid-19, contratación pública estratégica.

\section{A PUBLIC CONTRACTS LAW \\ FOR TIMES OF CRISIS}

\section{Abstract}

This paper analyzes how Spanish public procurement laws has been able to face crisis situations throughout history, highlighting the difficulties of Law 9/2017 to respond to the health and economic crisis situation caused by the covid-19. This requires strategic public procurement with the capacity to respond to future crisis situations.

KEYwords: public contracts; health and economic crisis, covid-19, strategic public procurement. 


\section{PRIMERA ETAPA: DE LA LEY DE CONTRATOS DEL ESTADO DE 1965 A LA LEY DE CONTRATOS DE LAS ADMINISTRACIONES PÚBLICAS DE 1995}

En el año que acaba de finalizar fue el 55 aniversario de la aprobación del Texto Articulado de la Ley de Contratos del Estado de 1965. La que fuera la primera Ley de contratación pública estaba integrada por la entonces nada desdeñable cifra de 125 artículos, 3 disposiciones adicionales y 7 disposiciones finales. Además de consagrar la tesis de la sustantividad del contrato administrativo (en un intento de poner fin a las disputas doctrinales del momento), esta Ley tendría el acierto de agrupar en un único texto las normas de contratación pública aplicables a todos los departamentos ministeriales, superando la diversidad de disposiciones existentes (como años antes había hecho la Ley de procedimiento administrativo de 1958) ${ }^{1}$. De este modo, se imponía un régimen jurídico administrativo que prestaba atención no sólo a la adjudicación de los contratos (como venía sucediendo hasta esa fecha), sino también a su gestación y ejecución ${ }^{2}$. Esta regulación se completó con el Reglamento General de Contratación de 1967, que contaba con 398 artículos, 9 disposiciones finales y una larga disposición derogatoria; dotando de flexibilidad al sistema normativo (por su parte, el reglamento de contratación de 1953 cumplía idéntica función para las entidades locales).

Como se recogía en la exposición de motivos de la Ley de bases de 1963, germen del Texto Articulado de 1965, ya en ese momento la contratación pública se concebía como «un instrumento decisivo para el desenvolvimiento de la acción social», transformándose en "pieza maestra del nuevo estilo de operar administrativo»; al tiempo que se intentaba que el Estado contratase «siempre de la forma más ventajosa y segura».

* Texto de la ponencia impartida en el Seminario sobre El impacto de la crisis en la legislación administrativa, organizado por el Área de Derecho Administrativo de la Universidad de La Laguna, en enero de 2021.

${ }^{1}$ Como señala Ariño (2002, 34-35), la abundante normativa anterior, constituida básicamente por los pliegos de condiciones generales, no constituía propiamente un derecho objetivo de la contratación pública, sino que era más bien una legislación procedimental y rituaria, preocupada exclusivamente por contratar a un precio lo más bajo posible; nos recuerda también este autor que la primera legislación de contratos (Ley de Bases de 1963 y Texto Articulado de 1965) fue obra de dos abogados del Estado, Martínez Esteruelas y Monedero Gil (poco hemos sabido después de la autoría de las leyes que le han sucedido en el tiempo).

${ }^{2}$ La gran virtud de la Ley de Contratos de 1965 fue codificar en un solo texto la regulación del contrato de obras públicas, hasta entonces dispersa y asistemática (VILlar Palasí y Villar Ezcurra, 1983, 47). A pesar de todo fue objeto de una severa crítica, por su «afán excesivamente innovador" y por el uso abusivo de «expresiones pseudotécnicas que oscurecen notablemente su contenido", así como por el «intento de regulación omnicomprensiva de todos los contratos del Estado» (Parada VÁzquez, 1965, 419-420). Vista con la perspectiva del tiempo, qué se podría decir ahora sobre la vigente legislación contractual. 
El legislador se enfrentó al difícil dilema de compatibilizar «agilidad y garantía», compensando las necesarias simplificaciones con un mayor rigor en la actividad de los órganos contratantes.

En esa época, términos como "conflicto de intereses», "fraude», «favoritismo» o «corrupción» no formaban parte del leguaje del legislador. Los problemas eran otros (si se quiere más básicos): de una parte, impedir que la Administración pudiera comprometer sus intereses con un empresario que no dispusiera de medios técnicos y financieros suficientes para cumplir con sus obligaciones, y, de otra, evitar que el contratista pudiera adquirir compromisos desproporcionados que perjudicaran el buen éxito del contrato.

La Ley de Contratos de 1965, con algunas modificaciones (las más importantes en 1973, dando lugar a un nuevo reglamento de contratación en 1975, y en 1986, con motivo de la incorporación a la UE), permitió a la Administración contratar la ejecución de obras, la prestación de servicios y la compra de suministros durante 30 años, hasta su derogación por la Ley de Contratos de las Administraciones Públicas de 1995 (superando situaciones como la crisis del petróleo de 1973 o la generada por la guerra del Golfo de 1990). Entre los objetivos perseguidos hay dos que siguen siendo de actualidad, como son «la necesidad de garantizar plenamente la transparencia de la contratación administrativa como medio para lograr la objetividad de la actividad administrativa y el respeto a los principios de igualdad, no discriminación y libre concurrencia»; y «la simplificación del procedimiento de contratación, suprimiendo algunos trámites que se han considerado menos necesarios y estableciendo preceptos concretos que tienden a lograr la indicada simplificación». Parece que estuviéramos hablando de la reforma de 2017 si no fuera porque el legislador de 1995 apostó entonces por la "potenciación de los contratos menores».

Desde el punto de vista de su contenido, la Ley de contratos de 1995 presentaba una estructura bipolar, que diferenciaba entre una "parte general», aplicable a todos los contratos; $y$ una "parte especial», en la que se recogían las peculiaridades de régimen jurídico de los contratos administrativos típicos. Sus 219 artículos se completaban con 9 disposiciones adicionales, 9 disposiciones transitorias y 4 disposiciones finales. Era una ley sencilla, que se asentaba sobre la distinción entre contratos administrativos y contratos privados, lo que tenía el inconveniente de situar en un ámbito de indefinición el régimen aplicable a los contratos celebrados por las empresas públicas y por otros entes instrumentales de naturaleza privada. Ese intento de eludir la aplicación del régimen de la contratación pública sería la causa de importantes críticas (Baño León las calificó de "piruetas jurídicas» ${ }^{3}$ y, en última instancia, de su posterior derogación (como reconoció la exposición de motivos de la Ley de contratos de 2007). Además, la primera ley de contratos de la democracia inició el proceso de elevar de rango algunos preceptos del Reglamento General de Contratación, introduciendo mayor rigidez en el sistema normativo.

3 Baño León, J.M.a a 2000, 15. 


\section{SEGUNDA ETAPA: DEL TEXTO REFUNDIDO DE 2000 AL TEXTO REFUNDIDO DE 2011}

La historia posterior resulta más conocida. La Ley 13/1995 tuvo una vigencia efímera (la más fugaz de todas) debido a sus numerosas modificaciones, que afectaron a 129 artículos (la más importante en 1999), lo que llevó a su derogación por el Texto Refundido de la Ley de Contratos de las Administraciones Públicas de 2000, que a su vez sería derogado por la Ley de Contratos del Sector Público de 2007 (desarrollada parcialmente por el Real Decreto 817/2009). El cambio de denominación abre paso a una nueva generación de leyes reguladoras de la contratación pública.

La Ley de contratos de 2007, aun teniendo como motivo determinante la trasposición de la Directiva 2004/18/CE, apostó por una reforma global, que se consolidó con la aprobación del Texto Refundido de la Ley de Contratos del Sector Público de 2011. Esta nueva regulación abordaba la actividad contractual pública de acuerdo con una definición funcional más acorde con la normativa europea, extendiendo su aplicación a todos los sujetos del sector público (rompiendo así con la estructura bipolar de la Ley de 1995).

El resultado fue una regulación densa, que dejaba poco espacio para el desarrollo reglamentario (el TR de 2011 constaba de 334 artículos, 36 disposiciones adicionales, 10 disposiciones transitorias, 6 disposiciones finales y 3 anexos), de difícil interpretación y aplicación. Ello motivó duras críticas por parte de la doctrina, sobre la que se llegó a decir que «jamás ha existido una Ley administrativa general tan confusa, tan mal redactada y compleja» (Ramón Parada ${ }^{4}$ ).

Entre sus objetivos, vuelve a aparecer la necesidad de avanzar en la simplificación y racionalización de la compra pública, disminuyendo los costes y cargas para la entidad contratante y para los contratistas. La solución parece pasar por la «plena inserción de los medios electrónicos, informáticos y telemáticos en el ámbito de la contratación pública, a fin de hacer más fluidas y transparentes las relaciones entre los órganos de contratación y los operadores económicos» (lo que conlleva una barrera de acceso para muchas pymes). Estos objetivos se complementan con otras novedades, como la adopción de la terminología comunitaria de la contratación, la incorporación de cláusulas sociales y medioambientales (como criterios de valoración de las ofertas o como condiciones especiales de ejecución) o la regulación del nuevo recurso administrativo especial.

Sin embargo, la Ley de Contratos de 2007 mostró pronto sus debilidades. Esta norma fue una herramienta ineficaz para combatir los efectos de la crisis económico-financiera iniciada en 2007. Adoptada la decisión de combatir los efectos de la crisis mediante la ejecución de obra pública (los conocidos Plan E y Plan.Es Economía Sostenible), el Gobierno se vio obligado a soslayar la aplicación de esa Ley para poder ejecutar en plazo los proyectos aprobados, estableciendo en su lugar un régimen jurídico ad hoc, más flexible, que -entre otras medidas- venía a declarar

\footnotetext{
${ }^{4}$ Parada Vázquez, R., 212, 235.
} 
la urgencia de la tramitación, acortar los plazos de tramitación, suprimir requisitos documentales o eximir de la clasificación del contratista. Los procedimientos ordinarios de contratación dejaron patente su escasa operatividad (según el Tribunal de Cuentas, los dos procedimientos más utilizados fueron el negociado sin publicidad y la contratación menor: en el primer plan los porcentajes fueron superiores al $40 \%$ $y$ al 30\%, respectivamente; $y$ en el segundo plan, a la inversa).

De otra parte, la nueva regulación tampoco evitó los casos de corrupción que salpicaron toda la geografía nacional en esos años. Quedó claro que los problemas de corrupción (que los hay allí donde hay posibilidades de enriquecimiento fácil) no se combaten sólo con nuevas leyes, cada vez más complejas: mayor regulación no es sinónimo de menor corrupción. Con independencia de la posibilidad de introducir algunas medidas concretas (cabe recordar ahora el «Decálogo de reglas para prevenir la corrupción en los contratos públicos» elaborado por Gimeno Feliu'), la mejora de la regulación no es suficiente si -como sostiene Andrés Betancor ${ }^{6}$ - no se actúa sobre los incentivos, tanto los que incitan a la corrupción como aquellos otros que la disuaden (establecimiento de un sistema de denuncia anónima, reforzamiento de los controles internos, trazabilidad y transparencia de la toma de decisiones, mejora de los medios a disposición de los tribunales de justicia, etc.), y si -añadimos nosotros- no se logra una mayor profesionalización de la contratación pública, mejorando la cualificación de los servicios de contratación (como propone el último Informe anual de la Oficina Independiente de Regulación y Supervisión de la Contratación -OIReScon-, de diciembre de 2020, que recomienda crear itinerarios de especialización).

En definitiva, la reforma de 2007 dio lugar a una legislación más prolija en extensión (el TR de 2011 tenía 115 artículos y 27 disposiciones adicionales más que la Ley de 1995) y más compleja en contenido sin que ello se tradujera en una mejor gestión y en una menor corrupción.

\section{TERCERA Y ÚLTIMA ETAPA: LA LEY DE CONTRATOS DEL SECTOR PÚBLICO DE 2017}

Cuando se pensaba que no podía haber textos más largos, la vigente Ley de contratos del sector público de 2017 bate todos los récords, con sus 347 artículos (de los cuales 312 tienen total o parcialmente carácter básico), 59 disposiciones adicionales, 5 disposiciones transitorias, 16 disposiciones finales y 6 anexos. Esta norma pretende ser un verdadero código de contratación pública, con el inconveniente de la rigidez formal que le otorga su carácter legal (también lleva camino de superar el récord de modificaciones parciales, 11 hasta ahora).

\footnotetext{
5 Gimeno Feliu, J.M. ${ }^{a}, 2014,1-8$.

6 Betancor, A., 2014, 1-3.
} 
El motivo de la aprobación de la Ley fue la transposición de las Directivas de contratación pública de 2014. No obstante, el nuevo texto se elaboró en un clima social y político condicionado por numerosos casos de corrupción, algunos muy mediáticos (como son los casos tres por ciento, Innova, Imelsa, Nóos, Púnica, Madeja, Palau, Gürtel, etc.). Se trata, por ello, de una ley que nace inspirada en la desconfianza (hacia los políticos, los funcionarios, los empresarios, los mecanismos de control...). Su redactor parece olvidar las decenas de miles de contratos que las Administraciones Públicas adjudican cada año: según datos del OIReScon, en 2019 se celebraron en nuestro país 129594 licitaciones públicas (28 283, correspondientes al sector público estatal; 34 925, al sector público autonómico; y 66 386, al sector público local). Esta desconfianza alcanza también a las empresas públicas y a los medios instrumentales, pero esto es otra historia que sería larga de contar.

A diferencia de reformas anteriores, los partidos políticos convierten la lucha contra la corrupción en uno de los objetivos de la nueva regulación. Por vez primera se recoge la obligación de los órganos de contratación de adoptar las «medidas adecuadas para luchar contra el fraude, el favoritismo y la corrupción, y prevenir, detectar y solucionar de modo efectivo los conflictos de intereses que puedan surgir en los procedimientos de licitación». También se exige que el informe anual de la OIReScon incorpore toda la información relacionada con la prevención, detección y notificación adecuada de los casos de fraude, corrupción, conflicto de intereses y otras irregularidades graves en la contratación 7 . De igual manera, la Estrategia Nacional de Contratación Pública deberá incorporar medidas que permitan combatir la corrupción. Esto parece una verdadera «declaración legal de culpabilidad ${ }^{8}$.

En esta línea, la medida estrella de la nueva Ley fue la supresión del procedimiento negociado sin publicidad por razón de la cuantía y la restricción de la contratación menor (que había sido el blanco de todas las críticas); así como la creación, como mecanismos alternativos, del procedimiento abierto simplificado y supersimplificado (abreviado o sumario). Medidas que fueron consecuencia de la transacción de las enmiendas n..$^{\circ} 125$ y 583 presentadas por los grupos parlamentarios de la oposición.

Se puede decir, sin temor a equivocarnos, que en este aspecto la reforma no ha cumplido sus objetivos. No sólo porque no ha logrado reducir el número de contratos menores y evitar el fraccionamiento indebido de los contratos (la Administración encontró en los anticipos de caja fija una solución parcial), sino especialmente porque los mismos grupos parlamentarios que promulgaron el cambio, una vez en el

\footnotetext{
${ }^{7}$ Es el módulo IV del informe anual de 2021, que tiene como título «la prevensión y lucha contra la corrupción en la contratación pública».

${ }^{8}$ La nueva Ley refuerza la visión hacendística de la contratación pública que, como apunta Santamaría Pastor, se incorpora ya en los reglamentos de 1967 y 1975, lo que responde al afán de control del Ministerio de Hacienda sobre los departamentos inversores (a los que siempre ha considerado «derrochadores impenitentes y tendencialmente corruptos») y a la desconfianza visceral sobre el contratista tipo, al que ve como «un pirata despachado», sólo atento «a captar fondos públicos para su negocio" $(2013,32)$.
} 
Gobierno, han modificado ambas regulaciones. Por lo que se refiere a la regulación de la contratación menor contenida en el artículo 118 (cuya interpretación provocó la división de las juntas consultivas) ${ }^{9}$, el Real Decreto-ley 3/2020 alteró su redacción, optando por la tesis más flexible a su utilización (de forma provocativa, Julio González ${ }^{10}$ propone la derogación del precepto, al que califica como un "pequeño franquestein») $)^{11}$. De repente, lo que era malo dejó de serlo: ahora, el contrato menor es necesario para dinamizar la compra pública local y para favorecer a las pymes (la realidad es que siempre lo fue). Por otro lado, el procedimiento abierto simplificado ha sido modificado cuatro veces en un solo año (Reales Decretos-leyes 15/20 y 16/2020 y Leyes 3/2020 y 11/2020), lo que evidencia las dificultades de su aplicación.

\section{LAS DIFICULTADES DE LA LEY 9/2017 PARA RESPONDER A LA SITUACIÓN DE CRISIS SANITARIA Y ECONÓMICA PROVOCADA POR LA COVID-19. LA NECESIDAD DE LLEVAR A BUEN PUERTO EL PLAN DE RECUPERACIÓN, TRANSFORMA- CIÓN Y RESILIENCIA DE LA ECONOMÍA ESPAÑOLA}

Como en anteriores ocasiones, la situación de crisis sanitaria y económica provocada por la covid-19 ha vuelto a mostrar las graves deficiencias de la legislación de contratos.

En un primer momento, ante la falta de planificación y la necesidad de dar una respuesta inmediata a la situación de emergencia sanitaria, el Gobierno decidió anteponer el interés público (proteger a las personas) a los principios de la contratación pública. Es así que, frente a la recomendación de la Comisión Europea (que en abril de 2020 propuso utilizar el procedimiento negociado sin publicidad), el Real Decreto-ley 7/2020 (art. 16) permite la tramitación de emergencia de la contratación de los bienes o servicios precisos para hacer frente a la covid-19 (lo que lleva a

${ }^{9}$ La oscura redacción del artículo 118.3 LCSP motivó que, inicialmente, las juntas consultivas de contratación se dividieran en dos grupos: de un lado, las partidarias de una interpretación restrictiva (Informe JCCA de Aragón n. ${ }^{\circ}$ 3/2018, seguido también por las de Madrid y País Vasco); y de otro, más numeroso, aquellas otras que postulaban una interpretación más flexible del precepto (Informe JCCP del Estado n. ${ }^{\circ}$ 41/2017, seguidas también por las de Andalucía, Canarias, Cataluña, Comunidad Valenciana y Galicia). Esta última fue también la posición que adoptó la Instrucción de la OIReSCcon n. ${ }^{\circ} 1 / 2019$, de 28 de febrero (y posterior nota aclaratoria).

${ }^{10}$ González García, J., 2020, 1-3.

${ }_{11}$ Disposición adicional primera del Real Decreto-ley 3/2020, de 4 de febrero (que incorpora la redacción que tenía la D.A. 34 del decaído proyecto ley de Presupuestos Generales del Estado para el año 2019). Previamente, la Disposición final 44.2 de la Ley 6/2018, de 3 de julio, de Presupuestos Generales del Estado para el año 2018, estableció una excepción al régimen general para los contratos celebrados por los agentes públicos del Sistema Español de Ciencia, Tecnología e Innovación, con el fin de elevar el límite de los contratos menores de suministro o de servicios a 50000 euros (añadiendo una nueva Disposición adicional LCSP, la n. ${ }^{\circ} 54$, que sería modificada por la Disposición final 2. ${ }^{a}$ del Real Decreto-ley 3/2019, de 8 de febrero, de medidas urgentes en el ámbito de la Ciencia, la Tecnología, la Innovación y la Universidad). 
la Junta Consultiva de Contratación Pública del Estado y al OIReScon a elaborar sendos documentos interpretativos, recordando las garantías mínimas de publicidad y transparencia). No obstante, esta medida pronto se reveló insuficiente, por lo que - en menos de un mes y siempre por la vía de urgencia- el Gobierno modificó en dos ocasiones dicho precepto con la finalidad de introducir nuevas excepciones a la legislación de contratos (DF 6. ${ }^{a}$ del R.D.-ley 8/2020 y DF $2 .{ }^{a}$ del R.D.-ley 9/2020). En especial, destaca el establecimiento de unas reglas específicas para la contratación de material en el extranjero, que incluye la posibilidad de anticipar el pago a la realización de la prestación (lo que resulta trascendental dada la falta de proveedores en nuestro país y la exigencia de competir con otros países en la adquisición de material). La rúbrica del Capítulo V del Real Decreto-ley 7/2020 es elocuente: «medidas para la gestión eficiente de las Administraciones Públicas». ¿Significa esto un reconocimiento de que los procedimientos ordinarios no son eficientes y que los mecanismos de racionalización de la contratación no funcionan? La respuesta parece haberla dado ya el propio Gobierno (a quien, por cierto, hubo que recordarle que la emergencia no está reñida con la transparencia).

No menos relevante es la falta de coordinación entre las Administraciones Públicas. Tras un momento inicial de tensión entre el Gobierno Central y las Comunidades Autónomas debido a la escasez de material, en agosto de 2020, el Ministerio de Sanidad licitó el mayor acuerdo marco de adquisición centralizada de la historia, con el objeto de adquirir material sanitario y equipos de protección individual por un valor superior a los 2500 millones de euros. Dicho acuerdo vino a sustituir al publicado dos semanas antes, que se decidió no adjudicar para poder atender las peticiones de aquellas Comunidades Autónomas que solicitaron su adhesión con posterioridad al valorar el riesgo de desabastecimiento, lo que provocó un cierto retraso (los últimos 5 lotes se adjudicaron el 30 de noviembre de 2020, 9 meses después del inicio de la pandemia). De esta forma, las administraciones únicamente tendrán que firmar los contratos de suministro con las empresas adjudicatarias, sin necesidad de nueva licitación. A su vez, en julio de 2020, España se adhirió a la compra centralizada de vacunas realizada por la UE, que prevé una compra inicial de 200 millones de dosis a diferentes empresas farmacéuticas, lo que permitirá contrarrestar la posición dominante de los proveedores y garantizar el abastecimiento de todos los Estados miembros.

$\mathrm{Y}$, en un segundo momento, la imperiosa necesidad de comprometer los fondos de recuperación europeos (en especial, los 72200 millones de euros de transferencias del programa Next Generation EU correspondientes al periodo 20212023), ha llevado al Gobierno a excepcionar nuevamente la aplicación de la LCSP, estableciendo un régimen especial que permita ejecutar "de modo ágil y eficaz» los proyectos previstos en el Plan de Recuperación, Transformación y Resiliencia de la Economía española. La regulación contenida en el Capítulo III del Título IV del Real Decreto-ley 36/2020 insiste en la tramitación urgente, eleva los importes de los procedimientos abiertos simplificados (tanto ordinario como abreviado), simplifica el régimen de autorizaciones e informes a recabar, promueve la elaboración de pliegos-tipo, acorta los plazos para la interposición y pronunciamiento del recurso especial, matiza el riesgo operacional de las concesiones y flexibiliza los encargos a 
medios propios (cuya regulación se modifica también en la Ley 11/2020, de Presupuestos Generales del Estado, aprobada el mismo día) ${ }^{12}$.

Dejando a un lado el alcance de las medidas adoptadas (que voces autorizadas como la de Moreno Molina ${ }^{13}$ han calificado de insuficientes), interesa poner el acento en el espíritu de esta regulación y los objetivos que persigue. Según se especifica en la exposición de motivos y se recoge en el propio articulado, el Real Decreto-ley responde a la necesidad acuciante de agilizar los procedimientos, «simplificando sus trámites y eliminando cuellos de botella normativos, de manera que la ejecución del Plan de Recuperación, Transformación y Resiliencia pueda llevarse a cabo de la forma inmediata». A tal fin, «incorpora una serie de instrumentos de carácter general dirigidos a reducir las barreras normativas y administrativas, así como un conjunto de medidas de modernización de las administraciones públicas, que permitan una gestión más ágil y eficiente, para facilitar la absorción de los mencionados fondos»; debiendo la Administración guiar su actuación por los «principios de agilidad, celeridad, simplicidad y claridad en los procedimientos, procesos y ejecución de tareas». Pero ¿no son éstos principios que deben regir toda la actuación de la Administración, no sólo en tiempo de crisis? Al menos, así lo dispone la Ley 40/2015.

\section{REFLEXIÓN FINAL}

En fin, el breve espacio de tiempo transcurrido entre las dos últimas grandes crisis económicas nos hace pensar que estamos ante una tendencia que se reproducirá cada vez con mayor frecuencia. Parece evidente que, en ambas crisis, los procedimientos tradicionales han sucumbido frente al establecimiento de reglas especiales de contratación. Sin embargo, frente a estas soluciones contingentes, de excepcionalidad, la tutela de los bienes jurídicos a los que sirve la contratación pública hace indispensable impulsar una contratación pública estratégica, que al mismo tiempo

${ }_{12}$ Con posterioridad a la elaboración del presente texto, la Junta Consultiva de Contratación Pública del Estado elaboró la Instrucción de 11 de marzo de 2011, sobre la tramitación de urgencia de los procedimientos de licitación de los contratos que se vayan a financiar con fondos procedentes del Plan de Recuperación, Transformación y Resiliencia, en la que se afirma que no cabe efectuar una declaración ex lege de urgencia de todos los procedimientos de licitación de contratos financiados con cargo a los citados fondos, sino que ha de justificarse caso por caso, y que es imprescindible respetar las exigencias de los principios de no discriminación, igualdad de trato y proporcionalidad. Siendo esto así formalmente, en la práctica, la gravedad de la situación económica del país, que cerró el año 2020 con una caída del PIB del 11\% (la peor de la OCDE y la mayor desde la guerra civil, superando a la provocada por la segunda guerra mundial), con 755000 trabajadores en ERTE (expediente de regulación temporal de empleo), con 724000 nuevos desempleados (que sitúa las cifras de paro en más 3,8 millones de personas, a los que hay que sumar otro 1,5 millón de trabajadores en situación de subempleo; con una tasa de paro juvenil superior al $40 \%$, esto es, 20 puntos por encima de la media europea) y 360105 afiliados menos a la Seguridad Social, hace difícil pensar que no sea posible justificar la urgencia de la mayor parte de los contratos que se liciten.

${ }^{13}$ Moreno Molina, J.A., 2021, 1-7. 
sea eficiente y dinamizadora de la actividad económica, lo que exige conciliar adecuadamente los principios de concurrencia y transparencia con procedimientos eficaces, que primen la calidad de la prestación y den respuesta a las necesidades colectivas en plazos razonables.

Las dos crisis económicas vividas en los últimos años han puesto de manifiesto la necesidad de reforzar la proactividad, la coordinación y la transparencia. En este sentido, cabe recordar las palabras de Gimeno Feliu ${ }^{14}$, en el sentido de que «necesitamos una nueva cultura de contratación pública: responsable, abierta, innovadora, cooperativa, profesionalizada, tecnológica y transformadora. Una contratación pública estratégica y proactiva y no meramente reactiva, que ponga en valor la calidad de la prestación». Pero hay que añadir también que, sobre todo, debemos garantizar un régimen de contratación que permita dar respuesta a las apremiantes necesidades públicas y que actúe como dinamizador de un modelo económico más sostenible, inteligente y resiliente que nos prepare mejor para afrontar futuras crisis sanitarias, climáticas, alimentarias, tecnológicas o energéticas.

RECiBIDO: marzo de 2021; ACEPTADO: abril de 2021

${ }^{14}$ Gimeno Feliu, J.M. ${ }^{a}$, 2020, 24; y 2021, 18. 


\section{BIBLIOGRAFÍA CITADA}

Ariño, G., Comentario a la Ley de Contrato de las Administraciones Públicas, Comares, 2002.

Baño León, J.M.a «La influencia del Derecho Comunitario en la interpretación de la Ley de Contratos de las Administraciones Públicas», Revista de Administración Pública, n. ${ }^{\circ}$ 151, 2000, p. 15.

Betancor, A., "Corrupción y contratación pública», Horror Vacui, Blogs Expansión, 3 de noviembre de 2014, disponible en https://www.expansion.com/blogs/horror-vacui.

Gimeno Feliu, J.M. ${ }^{a}$, «Decálogo de Reglas para prevenir la corrupción en los Contratos Públicos», Observatorio de Contratación Pública, 12 de noviembre de 2014, disponible en http:// www.obcp.es/.

Gimeno Feliu, J.M. ${ }^{a}$, «La crisis sanitaria covid-19. Reflexiones sobre su incidencia en la contratación pública y las soluciones adoptadas», Observatorio de Contratación Pública, 22 de abril de 2020, disponible en http://www.obcp.es/.

Gimeno Feliu, J.M.a, «De las ideas a la acción en la gestión de los fondos europeos: reflexiones propositivas para el diseño de una adecuada gobernanza en su ejecución», Observatorio de Contratación Pública, 25 de enero de 2021, disponible en http://www.obcp.es/.

Gimeno Feliu, J.M. ${ }^{a}$, «De las ideas a la acción en la gestión de los fondos europeos: reflexiones propositivas para el diseño de una adecuada gobernanza en su ejecución», Observatorio de la Contratación Pública, 25 de enero de 2021, disponible en http://www.obcp.es/opiniones/ de-las-ideas-la-accion-en-la-gestion-de-los-fondos-europeos-reflexiones-propositivas-para.

Gimeno Feliu, J.M.a «La adquisición masiva de vacunas», El Cronista del Estado Social y Democrático de Derecho, n. ${ }^{\circ}$ 93-94, 2021.

González García, J., "Contratos menores: nuevo cambio legislativo», Global Politics and Law, 5 de febrero de 2020, disponible en https://www.globalpoliticsandlaw.com/.

Moreno Molina, J.A., «Las insuficientes medidas de simplificación y agilización de la contratación pública recogidas en el Real Decreto Ley 36/2020», Observatorio de Contratación Pública, 4 de enero de 2021, disponible en http://www.obcp.es/.

Parada Vázquez, R., «La nueva Ley de Contratos del Estado», Revista de Administración Pública (RAP), n. ${ }^{\circ} 47,1965$.

Parada Vázquez, R., Curso de Derecho Administrativo, t. I, 19. ${ }^{a}$ ed., Marcial Pons, 2012, p. 235.

Santamaría Pastor, J.A., «La constante e interminable reforma reforma de la normativa sobre contratación pública», Revista Española de Derecho Administrativo (REDA), n. ${ }^{\circ}$ 159, 2013 , p. 32.

Villar Palasí, J.L. y Villar Ezcurra, J.L., Principios de Derecho Administrativo, t. III, Contratación administrativa, Universidad Complutense, 1983. 
